\title{
Evaluation of the Effect of Various Concentration of Sodium Hypochlorite on the Surface Roughness of ProTaper Rotary Files Using Atomic Force Microscopy: An In Vitro Study
}

\author{
${ }^{1}$ Tina Anto, ${ }^{2}$ Archa Anil, ${ }^{3}$ Liza George, ${ }^{4}$ Prasanth Dhanapal, ${ }^{5}$ Charlie KM, ${ }^{6}$ Sinju Paul
}

\begin{abstract}
Aim: The aim of this study was to evaluate the effect of different concentrations of sodium hypochlorite $(\mathrm{NaOCl})$, solutions on the surfaces of nickel-titanium $(\mathrm{Ni}-\mathrm{Ti})$ rotary files using atomic force microscopy (AFM).
\end{abstract}

Materials and methods: Sixteen unused ProTaper Ni-TI files of size F1, were selected for AFM evaluation at apical, middle and coronal segments. The selected files were then assigned into 4 groups

- Group 1: Unused files $(n=4)$

- Group 2: $2.5 \%$ sodium hypochlorite irrigation $(n=4)$

- Group 3: $5.25 \%$ sodium hypochlorite irrigation $(n=4)$

- Group 4: $8.25 \%$ sodium hypochlorite irrigation $(n=4)$

The files were used as per the manufacturer's instruction, and the number of uses was restricted to five. Normal irrigation protocol was followed and the only variable being a concentration of sodium hypochlorite $(2.5 \%, 5.25 \%, 8.25 \%)$. The post-treatment roughness average (RA) and root mean square (RMS) of apical, middle and coronal segments of the scanned profiles were then recorded using AFM and compared with the initial readings. The data was tabulated and analyzed using one-way analysis of variance (ANOVA).

Conclusion: Within the limitations of the present study, it was concluded that different concentrations of sodium hypochlorite showed effect on the surface characteristics of $\mathrm{Ni}-\mathrm{Ti}$ instruments.

Keywords: Atomic force microscopy, Nickel-titanium files, Sodium hypochlorite, Surface roughness

How to cite this article: Anto T, Anil A, George L, Dhanapal P, Charlie KM, Paul S. Evaluation of the Effect of Various Concentration of Sodium Hypochlorite on the Surface Roughness of ProTaper Rotary Files Using Atomic Force Microscopy: An In Vitro Study. Cons Dent Endod J 2018;3(2):56-61.

Source of support: Nil

Conflict of interest: None

${ }^{1,2}$ Postgraduate Student, ${ }^{3} \mathrm{HOD},{ }^{4}$ Professor, ${ }^{5}$ Reader, ${ }^{6}$ Senior Lecturer

${ }^{1-6}$ Department of Conservative Dentistry and Endodontics, Annoor Dental College and Hospital, Ernakulam, Kerala, India

Corresponding Author: Tina Anto, Postgraduate Student, Department of Conservative Dentistry and Endodontics, Annoor Dental College and Hospital, Ernakulam, Kerala, India, e-mail: drtinasweety@gmail.com

\section{INTRODUCTION}

Nickel-titanium (Ni-Ti) alloys were developed in the early 1960s by Buehler WF, a metallurgist at the Naval OrdnanceLaboratory in Silver Springs, Maryland, USA. Later on, Walia et al.introduced Ni-Ti endodontic rotary files to the field of endodontics because of its property of superior elasticity and resistance to torsional fracture. ${ }^{1}$ Studies have shown that Ni-Ti instruments have superior shaping ability compared to stainless steel files. ${ }^{2}$

The endodontic files are manufactured with alloys containing approximately $56 \%$ (weight) nickel and $44 \%$ (weight) titanium. Even though Ni-Ti instruments have many advantages, the incidence of instrument separations and fractures are very common during clinical use. ${ }^{3}$ The instrument fracture can occur in two different ways: fracture because of torsional fatigue and fracture because of flexural fatigue. ${ }^{4}$

The cleaning and shaping procedures make the files in constant contact with the irrigants. Recent studies have shown that even short-term contact with many of the irrigants can cause alterations on the surface of Ni-Ti instruments. ${ }^{5}$ Sodium hypochlorite is the most commonly used endodontic irrigant whose antimicrobial effectiveness is based on its high $\mathrm{pH}(\mathrm{pH}>11)$. During clinical use and cleaning and sterilization procedures. There is constant contact between rotary $\mathrm{Ni}$-Ti instruments and the $\mathrm{NaOCl}$ solution which may lead to instrument separation. ${ }^{6}$

Recently many studies have investigated the surface alterations on the files using SEM evaluation, but it did not gain popularity because it requires sample preparation. AFM (5500-Agilent Technologies, USA), a recent examination technique is used to reconstruct three-dimensional surface topography images in real time. It belongs to the scanning probe microscopy family with a tip attached to a flexible cantilever which is used to examine the surface and probe the sample surface. Deflection if any occurs in the z-direction due to surface topography during tip scanning over the sample surface. A photodiode can detect this deflection through a laser beam focused on and reflected from the rear of the cantilever. Thus AFM can record data of the samples in digital form as sets of $\mathrm{x}, \mathrm{y}$ and $\mathrm{z}$ values. 
The aim of this study is to evaluate the effect of various concentrations of sodium hypochlorite on the surface of the used ProTaper file using AFM.

\section{MATERIALS AND METHODS}

A total of sixteen ProTaper F1 files (Dentsply mailefer) were used for this study. Various concentrations of sodium hypochlorite; $2.5 \%, 5.25 \%, 8.25 \%$ were used as irrigants for 40 seconds.Cleaning and shaping were done on the distal roots of mandibular molars using the standard irrigation protocol for about 2 minutes. The samples were then divided into four groups after five uses based on concentration of sodium hypochlorite used.

- Group 1: F1 files instrumented with 2.5\% sodium hypochlorite

- Group 2: F1 files instrumented with 5.25\% sodium hypochlorite

- Group 3: F1 files instrumented with $8.25 \%$ sodium hypochlorite

- Group 4: Unused F1 files (control)

After five uses on the distal root of mandibular molars, the specimens were thoroughly rinsed with $5 \mathrm{~mL}$ of normal saline and then dried with filter paper. Specimens were attached to the metal base of AFM (5500-Agilent Technologies, USA) with double-sided tape. Sixteen surface areas along $3 \mathrm{~mm}$ sections at the tip of the files (perfect squares of $1 \mathrm{~mm} \times 1 \mathrm{~mm}$ )

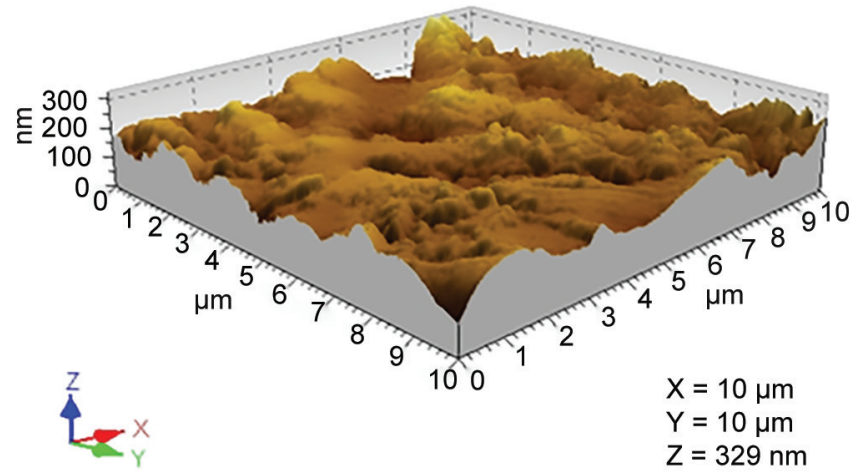

Fig.1: F1 files after instrumentation with $2.5 \%$ sodium hypochlorite

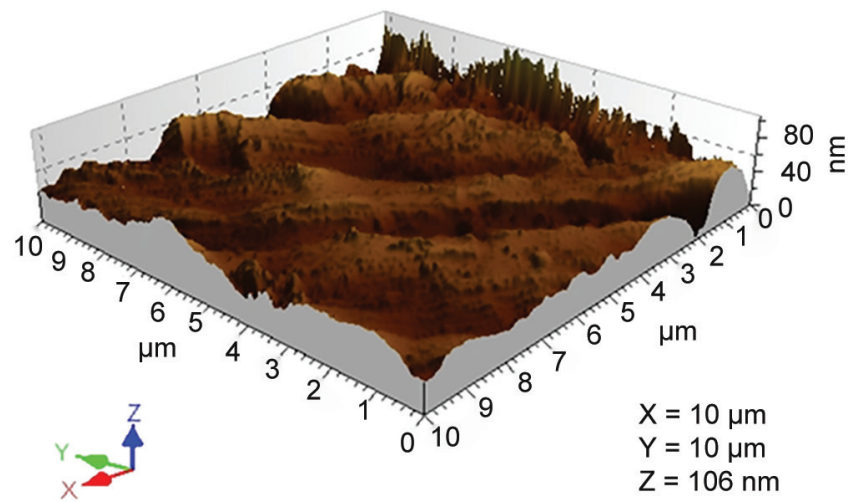

Fig. 3: F1 files after instrumentation with $8.25 \%$ sodium hypochlorite were analyzed by AFM operating in contact mode under ambient conditions. Three-dimensional images were processed using Gwyddion software. For all the four groups the root mean square value (RMS) of the scanned surface profiles was recorded (Figs 1 to 4 ), and data were analyzed using one-way ANOVA and Duncan's multiple range tests.

\section{RESULT}

The present study deals with testing whether there is any significant difference in the mean RMS values among the four groups. The four groups are- $2.5 \% \mathrm{NaOCl}, 5.25 \%$ $\mathrm{NaOCl}, 8.25 \% \mathrm{NaOCl}$ and control. One-way ANOVA is used for the analysis and post hoc test was done. Error bars are also drawn to visually understand the difference. In all the analysis significance level is taken to be 0.05 . Statistical analysis was carried out using statistical package, SPSS (version 22.0.0.0).

\section{Descriptive Statistics}

The descriptive statistics of the RMS values are obtained in Table 1.

From the descriptive statistics (Table 1) it can be observed that the mean RMS value is highest for $8.25 \%$ $\mathrm{NaOCl}$ group and the least for control group.

The box plot (Graph 1) suggests that the RMS values are distributed symmetrically and also contains no outliers.

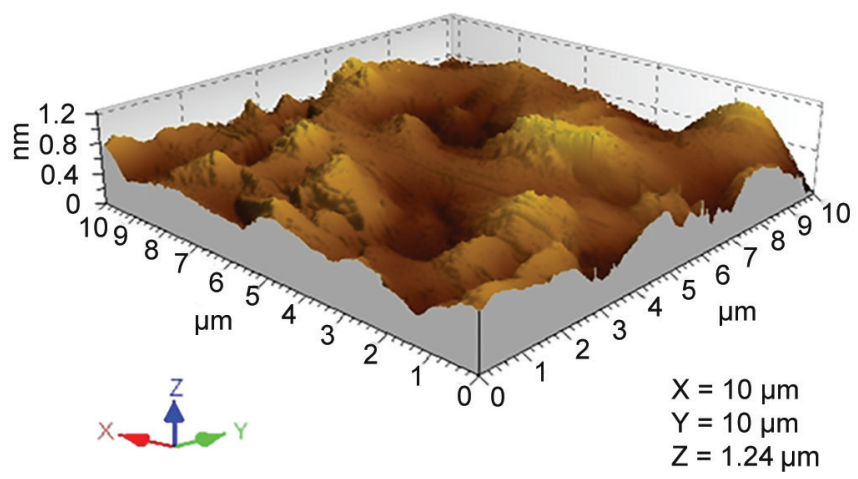

Fig. 2: F1 files after instrumentation with $5.25 \%$ sodium hypochlorite

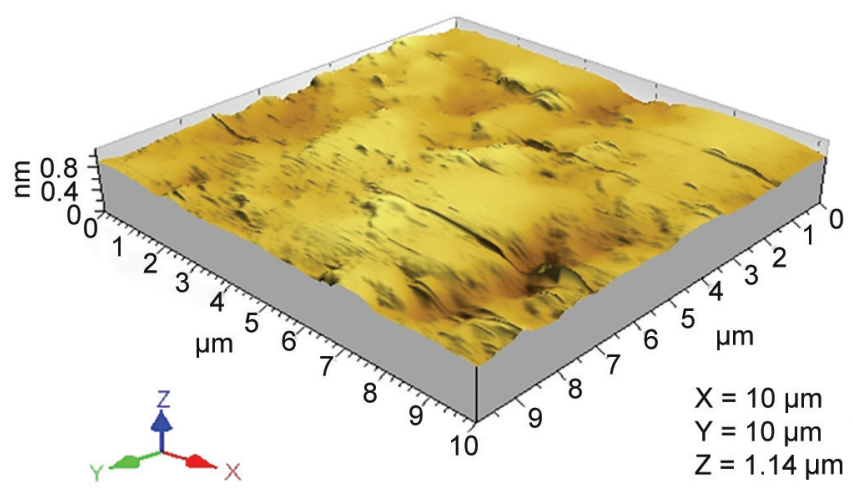

Fig. 4: Unused F1 files 


\section{Normality Test}

Before proceeding with the one-way ANOVA test, it is necessary that the assumptions of the test are satisfied. One of the assumptions is that the values are normally distributed for each group. Shapiro-Wilk's test is used to test the null hypothesis that the values are normally distributed.

Table 2 suggests that the values are normally distributed for each group. Another assumption is that the population variances among the groups should be equal. Levene's test is used to test the null hypothesis that the population variances among the groups are equal.

Table 1: Descriptive statistics

\begin{tabular}{lllll}
\hline Percentage (\%) & $N$ & Mean & Std. deviation & Std. error \\
\hline 2.5 & 4 & 2.765 & 0.129 & 0.064 \\
5.25 & 4 & 3.513 & 0.290 & 0.145 \\
8.25 & 4 & 4.113 & 0.085 & 0.043 \\
Control & 4 & 1.233 & 0.067 & 0.033 \\
\hline Total & 16 & 2.906 & 1.123 & 0.281 \\
\hline
\end{tabular}

Table 2: Test for normality

\begin{tabular}{llll}
\hline Percentage (\%) & Statistic & df & Significance \\
\hline $2.5 \%$ & 0.867 & 4 & 0.287 \\
$5.25 \%$ & 0.951 & 4 & 0.722 \\
$8.25 \%$ & 0.971 & 4 & 0.850 \\
Control & 0.995 & 4 & 0.983 \\
\hline
\end{tabular}

Table 3: Test for variance

\begin{tabular}{llll}
\hline Levene statistic & $d f 1$ & $d f 2$ & Significance \\
\hline 1.629 & 3 & 12 & 0.235 \\
\hline
\end{tabular}

Table 3 can be observed that the population variances among the groups are equal.

\section{One-way ANOVA}

As the assumptions of the test are satisfied, ANOVA is used to test the null hypothesis that there is no significant difference in mean RMS values among the groups (Table 4).

From Table 4, it can be concluded that there is significant difference in mean RMS values $[\mathrm{F}(3,12)]=220.665$, $\mathrm{p}$-value $<0.001$ ) among the groups. As there is significant difference among the groups, Tukey's post-hoc test was conducted.

Table 5 suggest that there is significant difference in mean RMS values among each pair of groups.

From the error bar (Graph 2) it can be observed that there is significant difference in mean RMS value among each group as there is no overlapping.

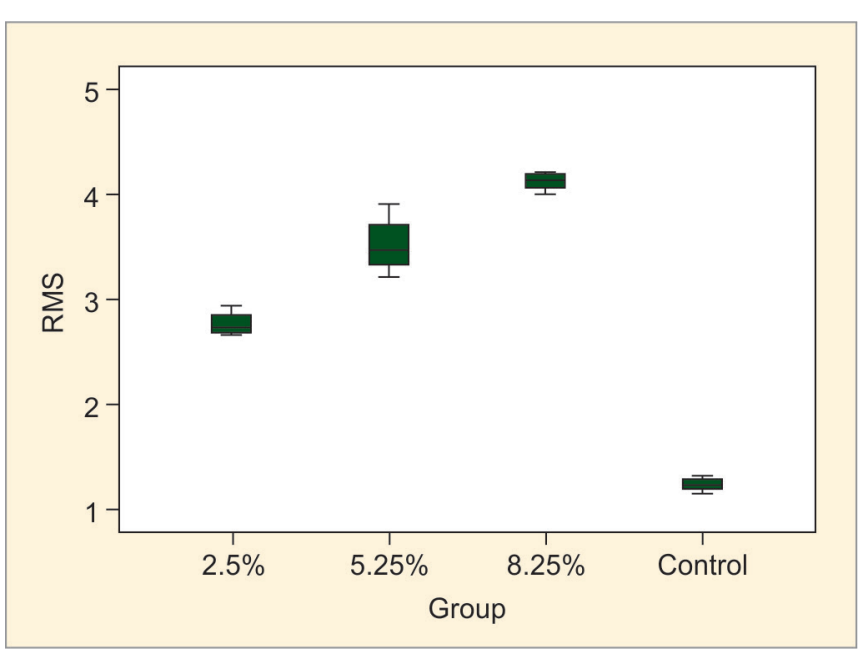

Graph 1: Box plot for RMS values

Table 4: One-way ANOVA

\begin{tabular}{llllll}
\hline & Sum of squares & $d f$ & Mean square & $F$ & Sig. \\
\hline Between groups & 18.576 & 3 & 6.192 & 220.665 & 0.000 \\
Within Groups & 0.337 & 12 & 0.028 & & \\
Total & 18.913 & 15 & & & \\
\hline
\end{tabular}

Table 5: Tukey's post-hoc test

\begin{tabular}{lllll}
\hline (I) Group & (J) Group & Mean difference (I-J) & Std. error & Significance \\
\hline \multirow{2}{*}{$2.5 \%$} & $5.25 \%$ & -0.748 & 0.118 & 0.000 \\
& $8.25 \%$ & -1.348 & 0.118 & 0.000 \\
\hline \multirow{3}{*}{$5.25 \%$} & Control & 1.533 & 0.118 & 0.000 \\
& $2.5 \%$ & 0.748 & 0.118 & 0.000 \\
\hline \multirow{3}{*}{$8.25 \%$} & $8.25 \%$ & -0.600 & 0.118 & 0.001 \\
& Control & 2.280 & 0.118 & 0.000 \\
\hline \multirow{3}{*}{ Control } & $2.5 \%$ & 1.348 & 0.118 & 0.000 \\
& $5.25 \%$ & 0.600 & 0.118 & 0.001 \\
& Control & 2.880 & 0.118 & 0.000 \\
\hline
\end{tabular}




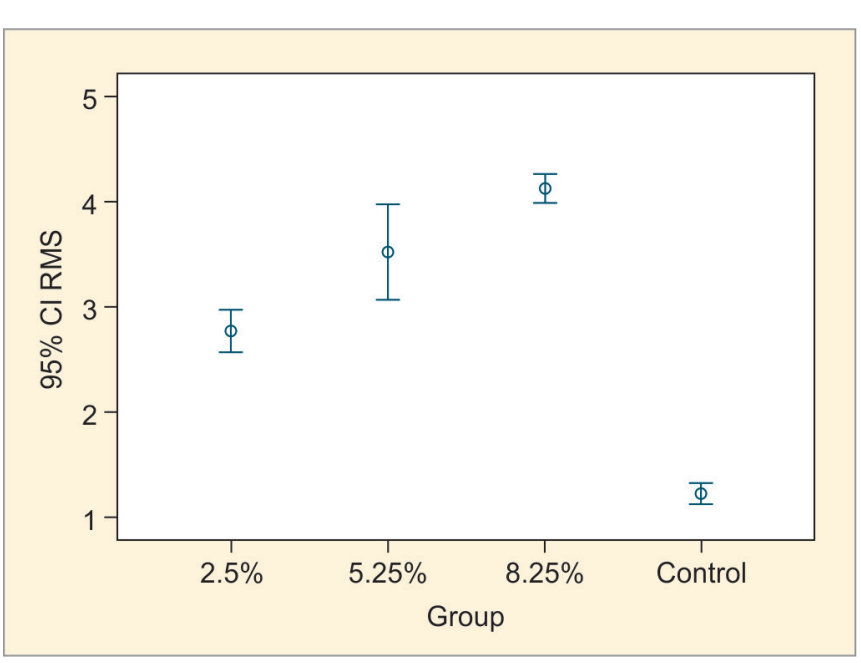

Graph 2: Error bar

\section{DISCUSSION}

The ideal endodontic irrigant should be antibacterial, dissolve the necrotic tissue, lubricate the canal, remove the smear layer and should not irritate the healthy tissues. Sodium hypochlorite is by far recognized as the most effective and efficient irrigant in endodontics. ${ }^{6}$ It is an effective antimicrobial with tissue-dissolving capabilities and has also been recommended as a cleaning agent for endodontic instruments after clinical use. ${ }^{7}$

Throughout the cleaning and shaping procedure and preparation, the endodontic irrigant is in constant contact with the endodontic files. Therefore, the effects of these solutions on the surface of instruments should be well taken into account. Previous studies have clearly stated that corrosion may be dependent on the time of contact and concentration of the irrigating solution. ${ }^{5}$ This corrosion, leading to surface alterations plays an important role in clinical instrument separation, and it is an undesired event that could affect the success of endodontic therapy. ${ }^{8}$ Recent studies have shown that sodium hypochlorite can corrode metal and alloys used in the manufacturing of endodontic files which causes, selective removal of nickel from the surface of $\mathrm{Ni}-\mathrm{Ti}$ instrument, leading to micro-pitting. ${ }^{9}$ These surface alterations can be best analyzed using SEM and AFM evaluation. Since scanning electron microscopy cannot give a qualitative data on surface irregularities, AFM was the chosen method of surface roughness evaluation in this study. ${ }^{10}$ Atomic force microscopy can provide an appropriate topographic contrast with greater details and will reconstruct a three-dimensional image of the sample surface topography in real time. ${ }^{11}$ Also AFM has ease of operation and user-friendly. Thus atomic force microscopy was recommended in this study to evaluate the surface characteristics of rotary Ni-Ti files. ${ }^{12,13}$

In accordance with the study done by the Topuz et al., $\mathrm{NaOCl}$ caused surface deteriorations, thereby contributing to the early fracture of $\mathrm{Ni}$-Ti files. ${ }^{12}$ Cavelleri et al., studied the effects of $\mathrm{NaOCl}$ on $\mathrm{Ni}$-Ti files surface using digital scanning microscopy and evaluated the surface roughness. ${ }^{14}$ The digital scanning microscopy evaluation demonstrated no alteration on the surfaces. This result may be attributed to the difference in the analysis method with our study.

In this study, ProTaper Ni-Ti file system used for the cleaning and shaping procedure of root canals were evaluated for surface alterations. The Nitinol alloy used in the manufacture of ProTaper instruments contains approximately $56 \%$ (wt.) nickel and $44 \%$ (wt.) titanium. ${ }^{15}$ The manufacturers of endodontic files, claim to have developed a technology with focus on altering the surface and alloy microstructure to enhance the efficacy and the longevity of the Ni-Ti instruments. ${ }^{16,17}$ Some manufacturers claim that electrochemical polishing of the surface of their Ni-Ti file systems can decrease corrosion. ${ }^{16}$ In this study AFM evaluation has shown that even unused ProTaper files have surface irregularities in a microscopic level which may be attributed to irregularities during alloy manufacturing.

In this study, it was observed that sodium hypochlorite at all concentrations caused deterioration on the $\mathrm{Ni}$ - $\mathrm{Ti}$ file surface. It is well documented that microstructural defects may lead to areas of stress concentration and crack formation which in turn weaken the structure of the Ni-Ti instrument. ${ }^{18}$ This is in accordance with the studies of Sonntag and Peters and Berutti et al. who observed localized corrosion defects and microcracks on the surfaces of $\mathrm{Ni}$-Ti files after immersion in 2.5 and $5 \% \mathrm{NaOCl}$ solution. ${ }^{19,20}$ In contrast to many previous studies, Cavalleri et al. reported that the contact of $\mathrm{NaOCl}$ for up to 10 minutes does not alter the surface of ProTaper files through corrosion. This may be attributed to the area of selection, and the method used for evaluation in the study. ${ }^{21}$ There are many previous studies proving the negative relationship between the concentration of $\mathrm{NaOCl}$ and surface roughness of Ni-Ti rotary files (Busslinger et al., Haikel et al., O'Hoy et al., Peters et al.).

The pulp dissolution property of $8.25 \% \mathrm{NaOCl}$ was significantly faster than any other tested concentration of $\mathrm{NaOCl}$ and had no effect on dentine flexural strength and modulus. ${ }^{22}$ in this study however increasing the concentration of $\mathrm{NaOCl}$ had a negative impact on the Ni-Ti files causing increased corrosion and micro-pitting which, in turn, can lead to early breakage during clinical use.

Recently various methods have been attempted to enhance the surface of Ni-Ti instruments and thus increase the surface hardness and flexibility and improve the resistance to cyclic fatigue and cutting efficiency of endodontic instruments have been advocated which 
includes plasma immersion ion implantation, thermal nitridation, cryogenic treatment, and electropolishing.

Plasma immersion ion implantation is a recent technique and was introduced by Conrad et al. and Tendys et al. It is facilitated by hot filament discharge, inductively coupled plasma, radio frequency, electron cyclotron resonance system or high voltage bias self-ignition. In this method, the specimen is first placed in an electric chamber and then immersed in the plasma. Following this then a highly negative pulsating voltage is applied to the sample. The plasma releases negative ions which are then extracted undergo acceleration and bombarded into the specimen. This can improve the mechanical properties of endodontic files such as hardness, friction coefficients, and wear and corrosion resistance.

In thermal nitridation, $\mathrm{Ni}$-Ti file samples are first annealed at $900^{\circ} \mathrm{C}$ for one and a half hours and then annealed at $1000^{\circ} \mathrm{C}$ for one hour in sealed containers. During this process, Nitrogen $(\mathrm{N})$ atoms are released and diffused into the samples. A steel foil attached to the walls of container, consisting of a notable amount of Chromium scavenge the atmospheric oxygen and makes the environment further reducing. Thus the surface consists of a thin outer layer of TiN and a thicker Ti2Ni layer underneath. This can considerably increase the mechanical property of the endodontic instrument. Shenhar et al. and Huang et al. have shown significantly increased the corrosion resistance of these treated files placed in contact with $5.25 \% \mathrm{NaOCl}$.

Another technique is cryogenic treatment, or the cold treatment of Ni-Ti files can improve the surface hardness and thermal stability of the metal. This treatment involves an optimum temperature range between $-60^{\circ} \mathrm{C}$ and $-80^{\circ}$ $\mathrm{C}$ depending upon the material of the sample and the quenching parameters used. The process involves submersing metal in a super-cooled bath containing liquid Nitrogen at very low temperature $\left(-196^{\circ} \mathrm{C} /-320^{\circ} \mathrm{F}\right)$ and then allowing the metal to slowly warm at room temperature.

Electropolishing is an electrochemical process for removal of the material layer from a metallic surface. It is usually employed as a final finish during manufacturing of Ni-Ti instruments. The surface chemistry and morphology are altered while surface imperfections are removed as dissolved metal ions. Simultaneously, titanium is oxidized to $\mathrm{TiO}_{2}$, which protects the underlying material from further corrosion.

$\mathrm{NaOCl}$ at all concentrations showed alterations on the surface of ProTaper files and because the lower concentration demonstrated fewer surface alterations, the use of lowest concentration may be recommended to avoid weakening of the ProTaper file.

\section{CONCLUSION}

Within the limitations of my study, it was shown that short-term contact between $\mathrm{NaOCl}$ and ProTaper instruments increased their RMS value indicating deterioration on the surface

\section{REFERENCES}

1. Plotino G, Grande NM, Cordaro M, Testarelli L, Gambarini G. A review of cyclic fatigue testing of nickel-titanium rotary instruments. Journal of endodontics. 2009 Nov 1;35(11):14691476.

2. Pedullà E, Plotino G, Grande NM, Pappalardo A, Rapisarda E. Cyclic fatigue resistance of four nickel-titanium rotary instruments: a comparative study. Annali di stomatologia. 2012 Apr;3(2):59.

3. Goo HJ, Kwak SW, Ha JH, Pedullà E, Kim HC. Mechanical Properties of Various Heat-treated Nickel-titanium Rotary Instruments. Journal of endodontics. 2017 Nov 1;43(11):1872-1877.

4. Tsujimoto M, Irifune $Y$, Tsujimoto $Y$, Yamada S, Watanabe I, Hayashi Y. Comparison of conventional and new-generation nickel-titanium files in regard to their physical properties. Journal of endodontics. 2014 Nov 1;40(11):1824-1829.

5. Popović J, Radenković G, Gašić J, Mitić A, Nikolić M, Barac $\mathrm{R}$, Živković S. Ultrastructural analysis of the surface of endodontic instruments after immersion in irrigating solutions. Serbian Dental Journal. 2015 Dec 1;62(4):157-165.

6. Gokturk H, Ozkocak I, Buyukgebiz F, Demir O. Effectiveness of various irrigation protocols for the removal of calcium hydroxide from artificial standardized grooves. Journal of Applied Oral Science. 2017 Jun;25(3):290-298.

7. Mohammadi Z. Sodium hypochlorite in endodontics: an update review. International dental journal. 2008 Dec;58(6): 329-341.

8. Gonçalves LS, Rodrigues RCV, Andrade Junior CV, Soares RG, Vettore MV. The Effect of Sodium Hypochlorite and Chlorhexidine as Irrigant Solutions for Root Canal Disinfection: A Systematic Review of Clinical Trials. Journal of Endodontics [Internet]. Elsevier BV; 2016 Apr;42(4):527-532.

9. Khasnis SA, Kar PP, Kamal A, Patil JD. Rotary science and its impact on instrument separation: A focused review. Journal of conservative dentistry: JCD. 2018 Mar;21(2):116-124.

10. Saghiri MA, García-Godoy F, Lotfi M, Mehrvazfar P, Aminsobhani M, Rezaie S. The effect of some fluids on surface oxidation and amount of released iron of stainless steel endodontic files. Scanning. 2012 Sep;34(5):309-315.

11. Caballero H, Rivera F, Salas H. Scanning electron microscopy of superficial defects in $\mathrm{T}$ wisted files and $\mathrm{R}$ eciproc nickel- $\mathrm{t}$ itanium files after use in extracted molars. International endodontic journal. 2015 Mar;48(3):229-235.

12. Fatma $Y$, Ozgur U. Evaluation of surface topography changes in three NiTi file systems using rotary and reciprocal motion: an atomic force microscopy study. Microscopy research and technique. 2014 Mar;77(3):177-182.

13. Fayyad DM, Mahran AH. Atomic force microscopic evaluation of nanostructure alterations of rotary $\mathrm{Ni} \mathrm{T}$ i instruments after immersion in irrigating solutions. International endodontic journal. 2014 Jun;47(6):567-573.

14. Valois CR, Silva LP, Azevedo RB. Atomic force microscopy study of stainless-steel and nickel-titanium files. Journal of endodontics. 2005 Dec 1;31(12):882-885. 
15. Cavalleri G, Cantatore G, Costa A, Grillenzoni M, Comin LC, Gerosa R. The corrosive effects of sodium hypochlorite on nickel-titanium endodontic instruments: assessment by digital scanning microscope. Minerva stomatologica. 2009 May;58(5):225-231.

16. Topçuoğlu HS, Topçuoğlu G, Akti A, Düzgün S. In vitro comparison of cyclic fatigue resistance of ProTaper Next, HyFlex $\mathrm{CM}$, OneShape, and ProTaper Universal instruments in a canal with a double curvature. Journal of endodontics. 2016 Jun 1;42(6):969-971.

17. Gutmann JL, Gao Y. Alteration in the inherent metallic and surface properties of nickel-titanium root canal instruments to enhance performance, durability and safety: a focused review. International endodontic journal. 2012 Feb;45(2): 113-128.
18. Stokes PU. Effect of disinfecting solution on endodontic instrument. Int Endod J. 1999;34:512-517.

19. Sonntag D, Peters OA. Effect of prion decontamination protocols on nickel-titanium rotary surfaces. Journal of endodontics. 2007 Apr 1;33(4):442-446.

20. Berutti E, Angelini E, Rigolone M, Migliaretti G, Pasqualini D. Influence of sodium hypochlorite on fracture properties and corrosion of ProTaper Rotary instruments. International endodontic journal. 2006 Sep;39(9):693-699.

21. Basrani B, Haapasalo M. Update on endodontic irrigating solutions. Endodontic topics. 2012 Sep;27(1):74-102.

22. Sağlam BC, Koçak S, Koçak MM, Topuz Öz. Effects of irrigation solutions on the surface of protaper instruments: A microscopy study. Microscopy Research and Technique. Wiley; 2012 Jul 13;75(11):1534-1538. 